Special Issue of the 6th International Congress \& Exhibition (APMAS2016), Maslak, Istanbul, Turkey, June 1-3, 2016

\title{
Effects of the Wind Speed and the Material Emplacement on the Output Signal of PZT Piezoelectric Energy Harvester
}

\author{
F. DEMiR ${ }^{a, *}$ AND M. ANUTGAN ${ }^{b, c}$ \\ ${ }^{a}$ Karabuk University, Electronics Technology Department, 78050, Karabuk, Turkey \\ ${ }^{b}$ Karabuk University, Faculty of Technology, Mechatronics Engineering Department, 78050, Karabuk, Turkey \\ ${ }^{c}$ Karabuk University, Thin Film Coatings Laboratory, Materials Research and Development Center (MARGEM), \\ 78050, Karabuk, Turkey
}

\begin{abstract}
In this study the electrical signal produced from wind energy through a $\left(\mathrm{Pb}_{x} \mathrm{X}_{1-x}\right)\left(\mathrm{Zr}_{y} \mathrm{Ti}_{z} \mathrm{Y}_{1-y-z}\right)$ piezoelectric transducer is analyzed. The material is placed onto a metal frame at different positions and voltage outputs of each are compared at different wind flow speeds and load resistance values. The absorption of the wind energy is tested by connecting a polyurethane material in parallel and perpendicular to the wind flow direction. The use of that material with optimum emplacement condition is shown to increase the voltage output by at least two orders of magnitude, where the maximum voltage output obtained is $13 \mathrm{~V}$, and the maximum power is $338 \mu \mathrm{W}$.
\end{abstract}

DOI: 10.12693/APhysPolA.131.362

PACS/topics: 81.05.--t

\section{Introduction}

Research on wind power is one of the hot topics regarding the renewable and sustainable energy sources. Wind is a natural result of the Earth's inhomogeneous heat distribution and can be regarded as the flow of the air molecules from high pressure to low pressure regions. Depending on the geography, the kinetic energy of the wind may reach sufficient levels for clean energy applications, where the energy harvested from the wind energy is environment-friendly and it does not emit any harmful gasses or radioactive particles $[1,2]$. The traditional method for the conversion of the wind energy to electrical energy is to rotate various sized blades by the wind power to induce electromotive force by the Faraday's law. However, this method has some limitations such as the requirement of a certain level of wind velocity to operate, a high installation cost and a big investment in infrastructure [3, 4]. Piezoelectric materials that can convert mechanical energy to electrical energy can be used to harvest wind energy, as an alternative to wind turbines. Although the energy obtained from piezoelectric converter is low, it is enough to power integrated circuits and autonomous wireless sensor applications with low energy consumption. Piezoelectric converters are low cost and do not require so much investment.

The first study on the wind energy harvesting using piezoelectric materials was made by Priya et al. [5, 6]. They have designed a windmill with several piezoelectric bimorph structures wrapped around a shaft. Despite its complex structure, this windmill could not manage to produce the desired amount of electrical energy. Taylor et al. have designed a piezoelectric eel using soft and

*corresponding author; e-mail: fundademir@karabuk.edu.tr flexible polyviniylidene fluoride (PVDF) based piezo material $[7,8]$ and $\mathrm{Li}$ and Lipson have used this study to develop a piezo-leaf. These two studies form the basis of most studies in this area. These are piezo-tree and piezoturbine studies that aim to use piezo materials with different sizes and structures at different angles to improve electrical energy harvesting from the wind energy [9-12]. Results show that sensors and integrated circuits can be powered by piezo materials using wind energy [13].

There are some important factors to consider in order to maximize the electrical energy produced from wind energy. One of them is choosing the right material, corresponding to application area. For instance, material and its structure should be appropriate to the changing weather and operation conditions. Another point is to match the material resonance frequency to the operation frequency under the wind. Besides, deformation of the piezo material should also be taken into consideration $[4,14]$.

The conversion of the wind energy to the electrical energy using piezoelectricity takes place in three stages: (i) wind is directed to the piezo material, (ii) mechanical energy is converted to electrical energy by piezoelectric effect, (iii) electrical signal output is processed and stored as electrical energy [6, 15].

In this study, a fiber composite piezoelectric material is being bent by wind of various intensities and the produced electrical energy is measured. For this purpose, a PZT material is attached to a metal frame at different positions. The effects of an additional polyurethane material attached to change the wind exposure capacity of the piezo material are compared to find the optimum emplacement condition.

\section{Materials and equipment}

The piezoelectric transducer used in the study produces electrical energy by flexing. The chemical 
composition of the material includes lead, zirconium and titanium at different ratios and is commonly represented by the chemical formula $\left(\mathrm{Pb}_{x} \mathrm{X}_{1-x}\right)\left(\mathrm{Zr}_{y} \mathrm{Ti}_{z} \mathrm{Y}_{1-y-z}\right)$ $(\mathrm{PZT})$. The structure is also reinforced by some embedded fibers to produce a composite structure with low brittleness and high electricity production capacity.

In Fig. 1a, cross sectional view of the fiber composite PZT piezoelectric transducer is provided. The inlaid interdigitated electrodes are coated over the whole surface area in order to improve the charge collection efficiency, similar to a geometry used in solar cells. The active region of the material comprises the embedded PZT fibers, parallel to each other and extending along the length of the material. These fibers are covered by a polymer matrix as a mechanical support. Figure $1 b$ shows the mode of operation of the PZT material used in this work. Different from the mode where the polarization is induced in the direction of the strain $\left(\mathrm{d}_{33}\right)$, our material is polarized in a direction perpendicular to the strain $\left(\mathrm{d}_{31}\right)$, which makes it a flexible-type piezoelectric transducer.

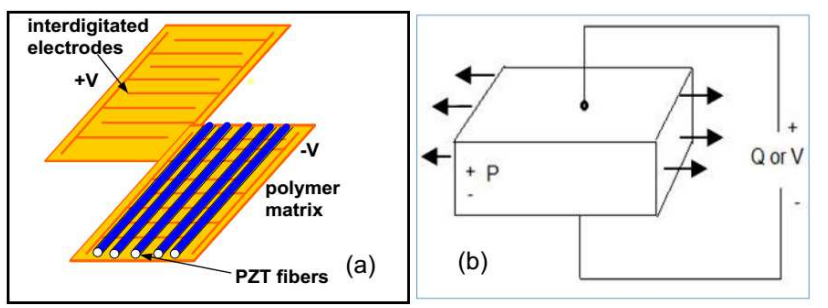

Fig. 1. Piezofiber material, (a) schematical cross section, (b) the electricity production mode $\left(\mathrm{d}_{31}\right)$.

\subsection{Experimental setup used in the study}

As shown in Fig. 2, a fan is used to form the wind, to move the PZT material. Fan speed is adjusted to the desired speed using a potentiometer and the wind velocity is read from an anemometer. The waveform of the produced electrical signal is very similar to sinus signal, which should be transferred to a DC storage medium. A bridge-type rectifier is preferred to rectify the produced signal. Signal is filtered through a capacitor after its

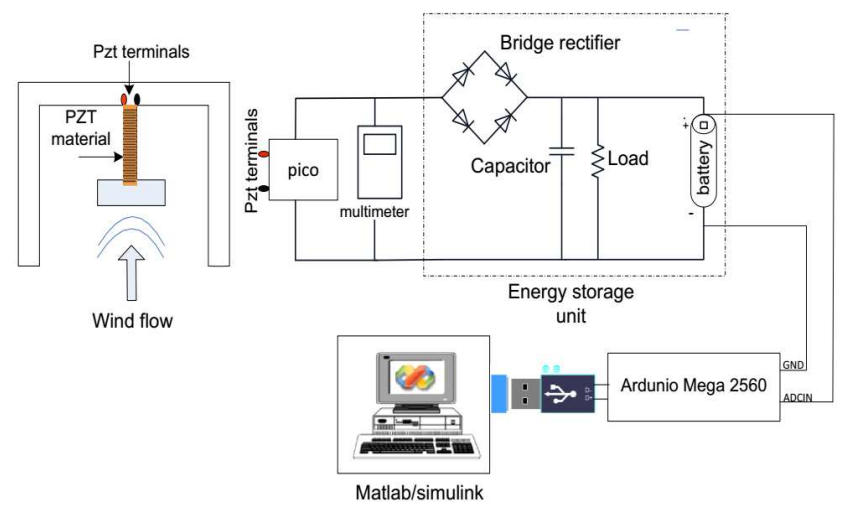

Fig. 2. Experimental setup used in the study. rectification, before sending it to the storage. This part of the experimental setup, which includes rectifier, capacitor, parallel resistor and battery, is called the energy storage unit. Battery charge versus time is monitored in real time and recorded in a computer using MATLAB. Analogue voltage of the battery is converted and sent to the computer as digital data with an Arduino development board. Voltage values, read and recorded through MATLAB, are also displayed on an alphanumeric LCD display attached to the Arduino development board.

\section{Results and discussion}

It is very important to determine how to position a PZT converter material to maximize the electrical energy produced from the wind energy. PZT material attached to a prepared metal frame can be adjusted and positioned at $x, y$ and $z$ axes to determine the best position relative to the wind direction (Fig. 3). After positioning the material on the axes, a polyurethane rectangular prism $\left(8 \times 12 \times 2 \mathrm{~cm}^{3}\right)$ is attached to the end of piezo material in parallel $(x 1, y 1, z 1$ in Fig. 3$)$ and perpendicular $(x 2, y 2$, $z 2$ ) directions. Piezo material without the polyurethane part is also used for comparison $(x 3, y 3, z 3)$.

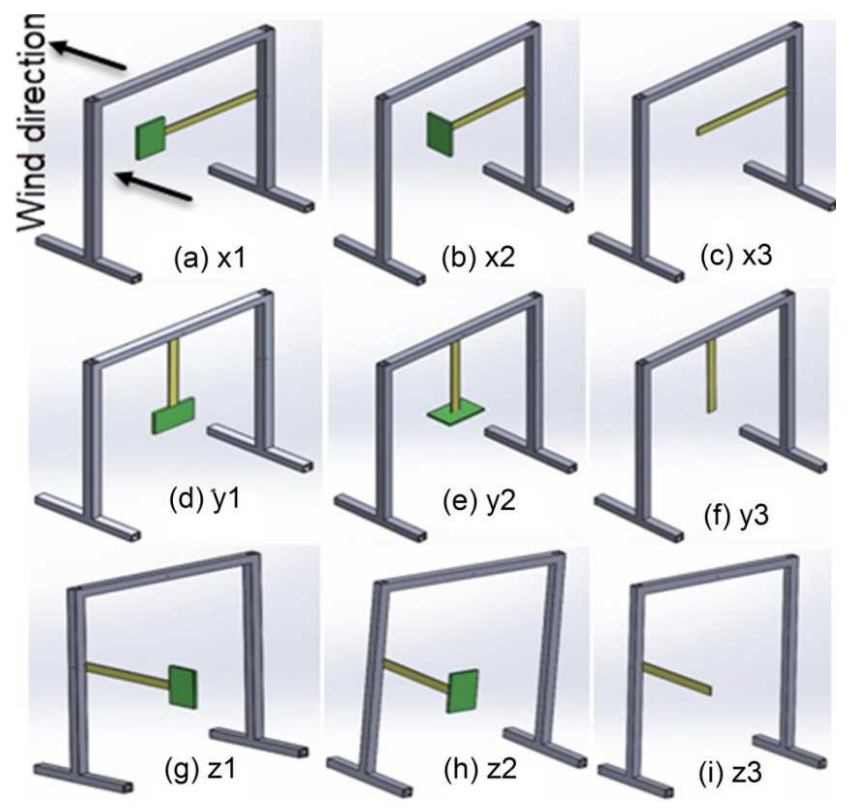

Fig. 3. Emplacements of the PZT material with respect to the wind directions, (a) $x 1$, (b) $x 2$, (c) $x 3$, (d) $y 1$, (e) $y 2$, (f) $y 3$, (g) $z 1$, (h) $z 2$, (i) $z 3$.

PZT fiber converter material is positioned as shown in Fig. 3 and $V_{\text {rms }}$ values are read on the load attached to the material. Load resistance, recommended for piezoelectric transducers, is calculated via

$$
R_{0}=1 /(2 \pi f C),
$$

where $f$ is the resonant frequency of the material and $C$ is its capacity. Capacitance of the PZT converter used in this study is $7-10 \mathrm{nF}$. Frequency varies from $32 \mathrm{~Hz}$ to $40 \mathrm{~Hz}$ according to the stress (i.e. the amount of flexing 
on the material). According to these values, parallel load to be attached for the maximum voltage output from the material should be around $350-400 \mathrm{k} \Omega$. Parallel loads used in the study are between 100 and $500 \mathrm{k} \Omega$ and the wind velocity is changed from 4 to $20 \mathrm{~m} / \mathrm{s}$. Figure 4 shows the $V_{\text {rms }}$ values obtained at each position for different load values with respect to the wind velocity.

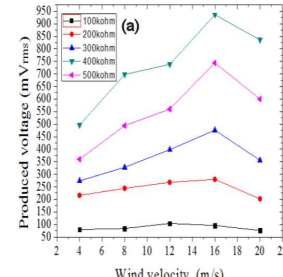
Wind velocity $(\mathrm{m} / \mathrm{s})$

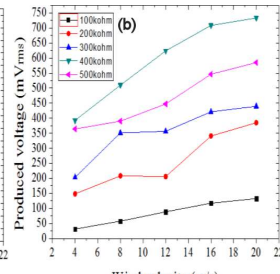
Wind velocity (m/s)
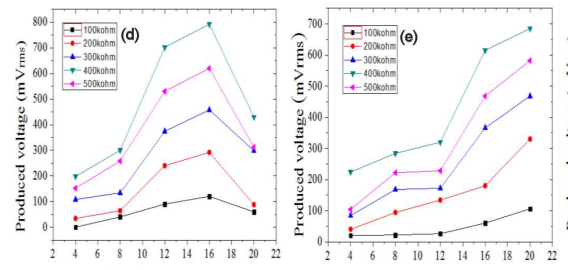
Wind velocity $(m / s)$
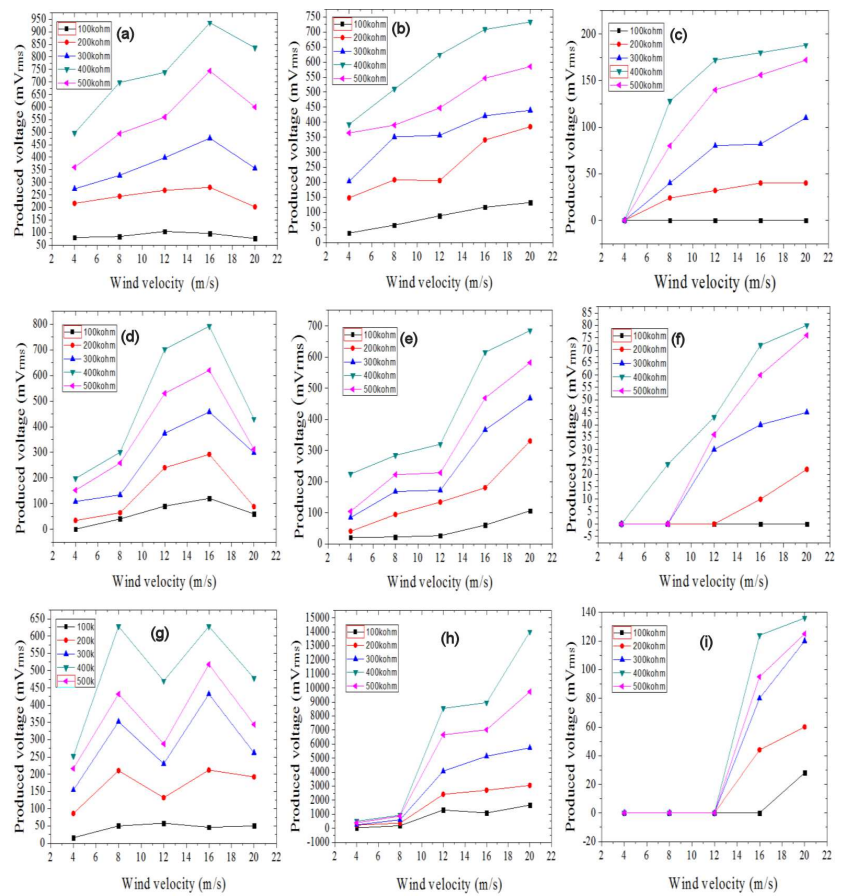
Wind velocity $(\mathrm{m} / \mathrm{s})$

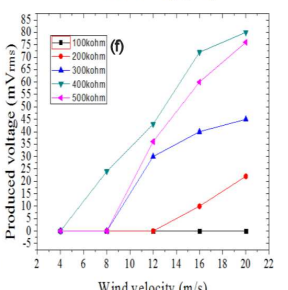
Wind velocity $(\mathrm{m} / \mathrm{s})$

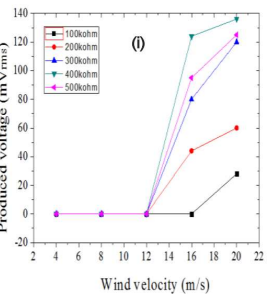

Fig. 4. $V_{\text {rms }}$ values for each position, for different load values, as functions of increasing wind velocity. (a) $x 1$, (b) $x 2$, (c) $x 3$, (d) $y 1$, (e) $y 2$, (f) $y 3$, (g) $z 1$, (h) $z 2$, (i) $z 3$.

According to the test results, the maximum $V_{\text {rms }}$ value obtained from the PZT converter material without using the polyurethane part is only $188 \mathrm{mV}$ ( $x 3$ in Fig. 3 and Fig. 4c). The maximum $V_{\text {rms }}$ values obtained by attaching the part as parallel and perpendicular are $936 \mathrm{mV}$ $(x 1)$ and $13000 \mathrm{mV}(z 2)$, respectively. Produced power is calculated to be $338 \mu \mathrm{W}$ by,

$$
P=V_{\mathrm{rms}}^{2} / R \text {. }
$$

Considering the results of the Fig. 4, there are two interesting notes to be mentioned. First, is the produced voltage output always directly proportional to the wind speed? It is obvious in the data of the emplacements $x 1$, $y 1$ and $z 1$ (Figure $4 \mathrm{a}, \mathrm{d}$ and $\mathrm{g}$, respectively) that the produced voltage has a tendency to decrease when the wind velocity reaches $16 \mathrm{~m} / \mathrm{s}$. It seems like there is a threshold wind velocity over which the air molecules have sufficient momenta to prevent the flexed PZT material from restoring its original position. It would be very similar if one holds a piece of paper and puffs on it continuously. Although the produced voltage output may be expected to be always proportional to the wind speed with a straightforward approach, the geometry of the energy harvester system must be well-designed to allow the oscillations with high amplitudes. This decreasing tendency of the voltage output is absent in the positions $x 2, y 2$ and $z 2$ (Fig. 4b, e and h, respectively) as can be inferred from the emplacements where the air resistance of the system decreases after it is flexed, allowing a continuous excitation-relaxation cycle. Throughout these measurements, the emplacement $z 2$ results in the highest measured $V_{\text {rms }}$ of $13000 \mathrm{mV}$. Note that, the decreasing behavior of the voltage output at the position $z 1$ at $12 \mathrm{~m} / \mathrm{s}$ wind speed is unclear at the moment.

Second interesting note for Fig. 4, is the difference in the voltage outputs for the emplacements of $x$ and $y$ (Fig. 4a, b, c and d, e, f, respectively). The cross sectional surface area of the energy harvesters facing the wind is the same for both $x$ and $y$. At the first glance, these emplacements might be expected to result in the same voltage outputs. On the contrary, this is not the observed case, where some portion of the wind energy in $y$ is transferred to the gravitational potential energy of the material.

\section{Conclusions}

In this study, a fiber-reinforced PZT piezoelectric transducer is bent by wind flow to produce electrical energy. The maximum energy is obtained from the piezo material when the wind flow manages to oscillate the material at some frequency, which is preferably close to the resonant frequency of that material. In addition, the surface area of the energy harvester must be designed so as to maximize the amount of wind it faces during excitation and to minimize it during relaxation. Also, it is shown that highest wind speed does not always guarantee the highest electrical signal output, since such strong wind levels have the potential to keep the PZT material more or less stationary in the excited state, which would avoid its relaxation, necessary for a vibrational energy harvester. That is why emplacement of the material against the wind and its geometry are important. Low current levels obtained from the material mean low capacity of power output, however these would be enough for low power consuming wireless sensors, biomedical sensors and integrated circuits, used in smart buildings if an increased number of materials are connected in series to each other. This kind of a power generation can be used as an alternative to wind turbines especially in urban areas, parks, gardens, roofs, bridges etc., where wind speed is not high enough and the place is not suitable for a wind turbine.

\section{Acknowledgments}

This work is financially supported by Scientific Research Projects Coordination Unit of Karabuk University (KBÜ-BAP-15/1-DR-004).

\section{References}

[1] E. Önal, R.Z. Yarbay, Istanbul Commerce Univ. J. Sci. 9, 77 (2010).

[2] H.N. Bayraç, J. Economy Soci. 1, 37 (2011). 
[3] M. Umeda, K. Nakamura, S. Ueha, Japan. J. Appl. Phys. 35, 3267 (1996).

[4] A. Şabanoviç, G. Çevik, M.F. Akşit, in 10th Int. Conf. Sustainable Energy Technologies 4-7 Sep., (2011).

[5] S. Priya, Appl. Phys. Lett. 87, 184101 (2005).

[6] S. Priya, C. Chen, D. Fye, J. Zahnd, Japan. J. Appl. Phys. 44, L104 (2005).

[7] G. Taylor, J. Burns, S.M. Kamman, W.B. Powers, T.R. Welsh, IEEE J. Oceanic Eng. 26, 539 (2001).

[8] J.J. Allen, A.J. Smits, J. Fluids Struct. 15, 629 (2001).

[9] H. Liu, S. Zhang, R. Kathiresan, T. Kobayashi, C. Lee, Appl. Phys. Lett. 100, 223905 (2012).
[10] X. Gao, W.H. Shih, W.Y. Shih, IEEE Trans. Ind. Electron. 60, 1116 (2013).

[11] S. Li, J. Yuan, H. Lipson, J. Appl. Phys. 109, 026104, (2011).

[12] S. Li, X. Shaanxi, H. Libson, Proc. ASME 2009 Conf. Smart Materials, Adaptive Structures and Intelligent Systems 1276, 611 (2009).

[13] S.N. Yun, Y.B. Ham, J.H. Park, Iccas-Sıce Aug, 5514 (2009).

[14] C. Luo, H.F. Hofmann, Energy Conversion Congress and Exposition (ECCE), IEEE, 2010, p. 4171.

[15] Y.K. Tan, S.K. Panda, 33rd Ann. Conf. IEEE Industrial Electronics Society (IECON), IEEE, 2007, p. 9. 